\title{
Preconception care for diabetic women for improving maternal and fetal outcomes: a systematic review and meta-analysis
}

\author{
Hayfaa A Wahabi*, Rasmeia A Alzeidan, Ghada A Bawazeer, Lubna A Alansari, Samia A Esmaeil
}

\begin{abstract}
Background: Preexisting diabetes mellitus is associated with increased risk for maternal and fetal adverse outcomes. Despite improvement in the access and quality of antenatal care recent population based studies demonstrating increased congenital abnormalities and perinatal mortality in diabetic mothers as compared to the background population. This systematic review was carried out to evaluate the effectiveness and safety of preconception care in improving maternal and fetal outcomes for women with preexisting diabetes mellitus.

Methods: We searched the following databases, MEDLINE, EMBASE, WEB OF SCIENCE, Cochrane Library, including the CENTRAL register of controlled trials and CINHAL up to December 2009, without language restriction, for any preconception care aiming at health promotion, glycemic control and screening and treatment of diabetes complications in women of reproductive age group with type I or type II diabetes. Study design were trials (randomized and non-randomized), cohort and case-control studies. Of the 1612 title scanned 44 full papers were retrieved of those 24 were included in this review. Twelve cohort studies at low and medium risk of bias, with 2502 women, were included in the meta-analysis.

Results: Meta-analysis suggested that preconception care is effective in reducing congenital malformation, RR 0.25 (95\% Cl 0.15-0.42), NNT17 (95\% Cl 14-24), preterm delivery, RR 0.70 (95\% Cl 0.55-0.90), NNT = 8 (95\% Cl 5-23) and perinatal mortality RR 0.35 (95\% Cl 0.15-0.82), NNT = 32 (95\% Cl 19-109). Preconception care lowers HbA1C in the first trimester of pregnancy by an average of $2.43 \%(95 \% \mathrm{Cl} 2.27-2.58)$. Women who received preconception care booked earlier for antenatal care by an average of 1.32 weeks (95\% Cl 1.23-1.40).
\end{abstract}

Conclusion: Preconception care is effective in reducing diabetes related congenital malformations, preterm delivery and maternal hyperglycemia in the first trimester of pregnancy.

\section{Background}

Diabetes mellitus (DM) is a global public health problem with expected 300 million diabetics by the year 2030 worldwide [1]. In many areas around the globe including the West as well as many developing and Middle Eastern countries, diabetes has become a major health burden affecting young adults and women in their reproductive age $[2,3]$.

Despite improved access and quality of antenatal care, women with pre-gestational diabetes and their fetuses are at increased risk of developing serious complications

\footnotetext{
* Correspondence: umlena@yahoo.com

Chair of Evidence-Based Healthcare and Knowledge Translation, King Saud University, Riyadh, Saudi Arabia
}

(c) 2010 Wahabi et al; licensee BioMed Central Ltd. This is an Open Access article distributed under the terms of the Creative Commons Attribution License (http://creativecommons.org/licenses/by/2.0), which permits unrestricted use, distribution, and reproduction in any medium, provided the original work is properly cited. compared with the non-diabetic pregnant women, including spontaneous abortion, preterm labor, hypertensive disorders, and delivery by cesarean section $[4,5]$. In the recent report of The Confidential Inquiry into Maternal and Child Health (CEMACH) from England, Wales and Northern Ireland, the perinatal mortality in mothers with type 1 and type $2 \mathrm{DM}$ is four times higher and the risk of congenital malformation in the babies of women with diabetes is nearly three times greater [4]. Similar reports from North America showed no significant improvement in fetal and neonatal outcomes of women with pre-gestational diabetes between 1988 and 2002 [6] despite the Saint Vincent Declaration in 1989 which sets a healthcare goal to improve the outcome of pregnancies in diabetic women [7]. 
Similar reports from the Middle East showed higher rate of perinatal mortality in diabetic as compared to non-diabetic women [8].

Many of the complications of DM during pregnancy can be prevented by optimizing maternal health in the preconception period. Glycemic control is one of the most important aspects of preconception care (PCC) [9]; however other aspects such as folic acid supplementation, smoking cessation, screening and treatment of diabetes complications and discontinuing teratogenic medication, are as important for improving maternal and fetal outcomes [10].

We carried out a systematic review to assess the effectiveness and safety of PCC in improving maternal and fetal outcomes for women with preexisting type 1 or type $2 \mathrm{DM}$.

\section{Methods \\ Type of studies}

We included in this review randomized trials (including cluster and quasi randomized studies) and cohort and case control studies, comparing the frequency of maternal and fetal adverse outcomes in diabetic women who received PCC with those who did not receive PCC.

\section{Type of participants}

Women of reproductive age with preexisting type 1 or type 2 diabetes mellitus who were not pregnant at the time of intervention.

\section{Type of intervention}

For the purpose of this review PCC is defined as the following either as sole intervention or in combination

1. Glycemic control by insulin and/or diet aiming at fasting blood glucose $\leq 5.7 \mathrm{mmol} / \mathrm{l}$ or/and postprandial blood glucose $\leq 7.8 \mathrm{mmol} / \mathrm{l}$ and/or glycosylated hemoglobin A (HbA1C) $\leq 7.0 \%)$

2 . Women counseling and/or education about diabetes complications during pregnancy, the importance of glycemic control and self monitoring of blood glucose level.

3. Preconception screening and treatment of complications of diabetes

4. The use of contraception until optimization of glycemic control is achieved

5. Intake of multivitamin or folic acid in the preconception period.

\section{Type of outcome}

\section{Maternal outcomes}

1. HbA1C level in the first trimester.
2. Gestation age at the time of the first visit to antenatal care clinic (booking visit).

3. Pregnancy complications including spontaneous abortion, termination of pregnancy due to congenital malformations, polyhydramninos, pre-eclampsia, preterm delivery (before 37 completed weeks from the last menstrual period) and induction of labour due to complication of diabetes.

4. Delivery by cesarean section or instrumental delivery.

5. Maternal hypoglycemia in the first trimester or any other adverse effect reported by the authors.

\section{Neonatal outcomes}

1. Congenital malformation related to maternal diabetes

2. Total mortality (stillbirth and neonatal death).

3. Birth trauma

4. Admission to neonatal intensive care unit (NICU).

5. Respiratory distress syndrome (RDS)

6. Macrosomia (birth weight $\geq 4 \mathrm{~kg}$ for term infants or birth weight $\geq 90^{\text {th }}$ percentile for the gestation age)

7. Small for gestational age (SGA) (birth weight below the $10^{\text {th }}$ percentile for the gestational age).

8. Shoulder dystocia.

\section{Exclusion criteria}

We excluded from this review reports which are not of comparative design and reports of conference proceedings or abstracts when there is no complete description of the trial or study.

\section{Search strategy}

The search strategy was developed in consultation of an information retrieval specialist. We searched the following databases, MEDLINE (1966-December 2009), EMBASE (1980-December2009), WEB OF SCIENCE (Science citation index-1970-December 2009), Cochrane Library up to the latest issue 2009, including the CENTRAL register of controlled trials and CINHAL (Cumulative Index to Nursing \& Allied Health 1982 -December 2009). (For full search strategy see Additional file 1: Appendix 1)

We reviewed the reference list of all relevant studies for any potential study not retrieved by the search strategy. Unpublished reports were not actively sought and there was no language limitation.

\section{Identification of included studies}

All titles and abstracts retrieved by the electronic search were screened independently by three reviewers and the studies which clearly did not meet the inclusion criteria were excluded. Copies of the full text of potentially 
relevant studies and trials were obtained and their eligibility was assessed independently by two reviews. Differences between reviewers were resolved by discussion or by consulting a third reviewer.

\section{Data extraction and studies assessment}

Three authors extracted data from the included studies using a designed form. The accuracy of the extracted data was checked by two other reviewers.

The Newcastle Ottawa Scale (NOS) was used for the assessment of cohort, case control studies and non-randomized trials [11]. Risk of bias in each study, was assigned according to the number of items on the NOS judged to be inadequate. We considered low risk of bias when one item is inadequate, medium risk of bias when up to three items are inadequate and high risk of bias when more than three items are inadequate. Data analysis was carried out with the use of Review Manager Software 5.0 (Cochrane Collaboration, Oxford, United Kingdom).

Meta-analysis was performed for studies with similar design and type of intervention, which we assessed to be at medium or low risk of bias using the fixed effect model. Heterogeneity is considered high when $\mathrm{I}^{2}>50 \%$ and explanation was attempted however subgroup analysis was not possible in most of the cases due to the small number of studies. Pooled data were presented as risk ratio (RR) with $95 \%$ confidence intervals (95\% CI) for dichotomous outcomes and as the means difference with $95 \%$ confidence intervals for continuous outcomes.

\section{Results}

The search retrieved 1612 potentially relevant titles of which the full papers of 44 relevant reports were reviewed (Figure 1). A total of 24 reports of 20 studies were included in this review [10,12-34]. (Three articles described the same cohort study with two interim $[17,18]$ and one final report [19], one study reported the outcomes for the same cohort in two articles $[10,29]$ and two articles report the outcomes of one cohort with one interim [31] and one final report [28]).

Twenty studies were excluded, 16 of them were excluded because they did not meet the inclusion criteria, 2 reports were of conference proceedings and in 2 studies data were not extractable (Additional file 1: Appendix 2).

Of the included studies, only one was a controlled trial, 11 studies were prospective cohort studies, 7 studies were retrospective cohort studies and one was a case control study (Tables 1-4).

\section{Assessment of the methodological quality of the included studies}

The cohort studies included in this review (Table1 \& 2) had adequate description of participants including description of some confounding factors such as the frequency of renal and vascular complications of diabetes between the PCC group and the control group. However all studies did not address the effect of the presence of confounding factors on the outcomes except for 2 reports which used regression analysis to evaluate the effectiveness of the PCC $[10,29]$.

In most of the cohort studies blinding of the control group was adequate because they were recruited after pregnancy when they attended for antenatal care, except for 2 studies [24,28], in which inadequate blinding of the control group cannot be excluded because they were informed about the importance of the PCC and were invited to attend. All participants received the same antenatal and post natal care except for one study [24] where participants were followed up in different health settings.

All cohort studies had adequate follow up for participants except for one study in which $52 \%$ of the PCC group were lost to follow up [26]. The assessors of the outcomes were not blinded to the participants' allocation except in one study [25].

Some of the studies at high risk of bias were initially designed to assess aspects of PCC other than its effectiveness in improving maternal and fetal outcomes, hence the poor methodological design when assessed with the NOS $[16,22,30]$.

PCC in all the cohort studies included control and self monitoring of blood glucose except for one which was designed to examine the effectiveness of preconception counseling on fetal and neonatal outcomes [30]. In addition to glycemic control, 4 studies included screening and treatment of complications of diabetes in the PCC program $[16,19,21,22]$. Only one cohort study (two reports) had comprehensive PCC program including, control and self monitoring of blood glucose, folic acid supplementation, smoking cessation advice and discontinuation of teratogenic drugs [10,29].

One case-control study was included in this review [13] (Table 3). It examined the effectiveness of multivitamin supplementation in the preconception period in preventing diabetes related congenital abnormalities. The study is at medium risk of bias due to possibility of recall bias during the interview of the mothers and the possibility that interviewers were not blinded to the outcome.

One trial was included in this review [12] (Table 4). The design of the trial was not clear as authors reported it as a randomized trial but the method for randomization was not described. There was no allocation concealment and lack of blinding introduced bias because both groups were aware of the importance of the glycemic control and the complications of diabetes during pregnancy. 


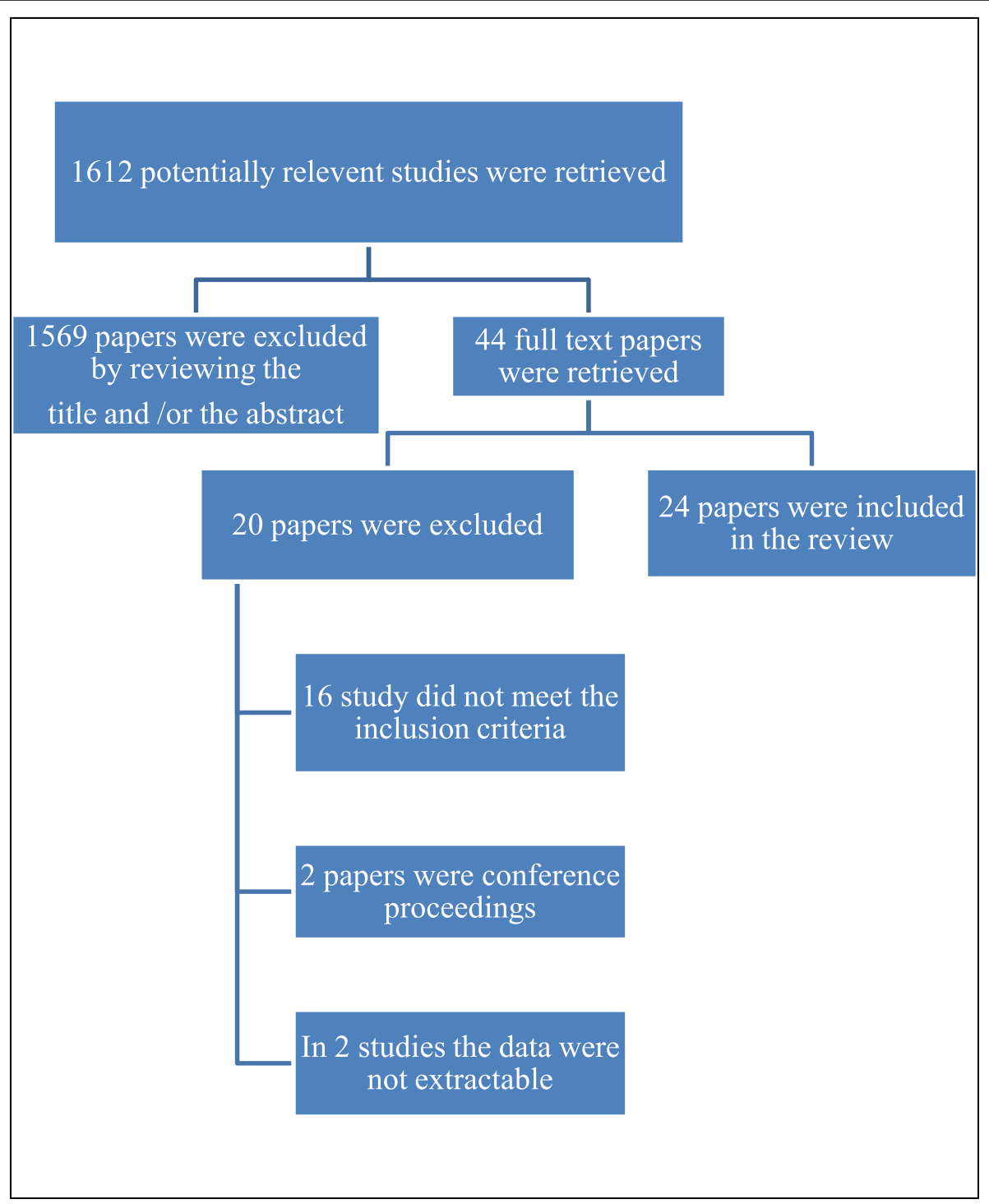

Figure 1 Process of selection of the studies for the systematic review.

Only 2 studies in this review evaluated maternal hypoglycemia, as an adverse effect of PCC [10,28].

\section{Outcome of PCC}

Similarity of participants, intervention, and outcomes in addition to the score of low or medium risk of bias, made meta-analysis possible for 12 cohort studies $[10,15,16,19-21,23,25,26,28,32,33]$ with 2502 participants (Tables 1, 2 \& 5 and Figures 2,3,4,5,6, and 7). Both dichotomous and continuous data were pooled but only when standard deviation and similar units were available for continuous data. Studies which were at high risk of bias or of a design other than cohort were excluded from the meta-analysis.
Meta-analysis suggested that preconception care is effective in reducing congenital malformation, RR 0.25 (95\% CI 0.15-0.42), NNT17 (95\% CI 14-24), preterm delivery, RR 0.70 (95\% CI $0.55-0.90), \mathrm{NNT}=8(95 \% \mathrm{CI}$ 5-23) and perinatal mortality RR 0.35 (95\% CI 0.15 $0.82), \mathrm{NNT}=32$ (95\% CI 19-109) (Figures 2, 3, and 4).

Meta-analysis of 5 trials show that PCC lowers $\mathrm{HbA} 1 \mathrm{C}$ in the first trimester of pregnancy by an average of $2.43 \%$ (95\% CI 2.27-2.58) and while there is high heterogeneity $\left(I^{2}=97 \%\right)$ this variation is in the size of the effect rather than the direction (Figure 5).

Women who received PCC booked earlier during pregnancy for antenatal care compared to women who did not, by an average of 1.32 week (95\% CI 1.4-1.23) (Figure 6) 
Table 1 Characteristics of included Prospective Cohort Studies

\begin{tabular}{|c|c|c|c|c|}
\hline $\begin{array}{l}\text { Study/Year } \\
\text { of } \\
\text { Publication } \\
\text { Reference } \\
\text { (country) }\end{array}$ & Participants & Intervention & Outcome & Risk of Bias (Notes) \\
\hline $\begin{array}{l}\text { Garcia- } \\
\text { Patterson } \\
1997[20] \\
\text { (Spain) }\end{array}$ & $\begin{array}{l}66 \text { participants with type I and } \\
\text { type II who attended the } \\
\text { preconception clinic and } 119 \\
\text { participants with type I and type } \\
\text { II diabetes who did not. }\end{array}$ & $\begin{array}{l}\text { PCC included intensive insulin } \\
\text { therapy, self-monitoring of } \\
\text { blood glucose and dietary } \\
\text { advice }\end{array}$ & $\begin{array}{l}\text { The HA1C was significantly } \\
\text { better in the PCC group than for } \\
\text { the NPCC group }(p=0.01) \text {. The } \\
\text { rate of cesarean section was } \\
\text { higher in the PCC group than } \\
\text { the NPCC. No differences were } \\
\text { observed in abortion, Pre- } \\
\text { eclampsia and preterm labor. } \\
\text { Small for gestation age was } \\
\text { more in the NPCC. }\end{array}$ & $\begin{array}{l}\text { Medium (The baseline } \\
\text { characteristics in relation to the } \\
\text { vasculopathy are different. No } \\
\text { blinding for the outcome } \\
\text { assessment). }\end{array}$ \\
\hline
\end{tabular}

Herman 24 women with type I diabetes

1999 [22] who attended the

(USA) preconception clinic, and 74 women with type I diabetes who did not attend the preconception clinic.
PCC included education, counseling, glycemic control, and assessment of complications of diabetes such as nephropathy and retinopathy

\section{Women who had PCC had}

significantly more spontaneous abortion, significantly lower level of $\mathrm{HA} 1 \mathrm{C}$ at booking and throughout pregnancy and significantly heavier infants at birth than NPCC group $(p<$ 0.05). There was no significant difference between the two groups in the frequency of infants with congenital malformations, gestation age at delivery or frequency of neonatal admission to the intensive care unit.

\begin{tabular}{ll}
\hline Jaffiol 2000 & 21 IDDM attended the pre- \\
[23] & conception care and 40 did not \\
(France) & attend
\end{tabular}

PCC included education, glycemic control self monitoring of blood glucose and Contraception

The investigated outcomes included polyhydramninos, preeclampsia, premature deliver, rate of cesarean section, rate of spontaneous and therapeutic abortion, perinatal and neonatal mortality, neonatal

hypoglycemia and birth trauma. Significant reduction in the total fetal loss, neonatal mortality and congenital malformations $(p<$ $0.05)$, the level of maternal HA1C in the $1^{\text {st }}$ trimester $(p<0.05)$ and total adverse obstetrics complications $(p<0.05)$

\begin{tabular}{lll}
\hline Jensen & 9 women with insulin & PCC included continuous \\
1986 [24] & dependent diabetes had & insulin infusion initiated 2 \\
(Denmark) & $\begin{array}{l}\text { preconception care and 11 } \\
\text { months prior to conception }\end{array}$
\end{tabular}
women with insulin dependent diabetic who did not receive preconception care.
No significant difference in congenital malformations and HA1C level, between the two groups
High (The study was not designed to assess the clinical outcomes of the preconception care but the differences in the socio-demographic features between the groups who attend the preconception care and those who did not. The target level for the glycemic control was not clear and the absolute level of $\mathrm{Hb} \mathrm{A1C}$ at booking and all through pregnancy for the study and the control groups was not mentioned)

Low (good report, clear intervention description, the comparative groups received same antenatal intervention. No blinding for outcome assessment)
High (small number of study and control group, many differences in the baseline characteristics in the severity of diabetes, 5 of the 11 control women were treated in the diabetic clinic in the hospital before pregnancy so they knew about the importance of glycemic control both groups have the same HA1C levels in early pregnancy)

\begin{tabular}{|c|c|c|c|c|}
\hline $\begin{array}{l}\text { Kitzmiller } \\
1991[25] \\
\text { (USA) }\end{array}$ & $\begin{array}{l}84 \text { women in preconception } \\
\text { care and } 110 \text { women had no } \\
\text { preconception care }\end{array}$ & $\begin{array}{l}\text { PCC included glycemic and } \\
\text { dietary control education, } \\
\text { exercise and contraception. }\end{array}$ & $\begin{array}{l}\text { The frequency of congenital } \\
\text { abnormalities in the PCC group } \\
\text { was } 1.2 \% \text { compared to } 10.9 \% \text { in } \\
\text { the NPCC group }(p<0.05) \text {. There } \\
\text { were } 12 \text { spontaneous abortion in } \\
\text { the preconception care group } \\
\text { and } 14 \text { in the group who } \\
\text { received no preconception care. }\end{array}$ & $\begin{array}{l}\text { Low (good report clear } \\
\text { methodology) }\end{array}$ \\
\hline
\end{tabular}


Table 1 Characteristics of included Prospective Cohort Studies (Continued)

\begin{tabular}{lll}
\hline Rosenn & 28 women in the preconception & PCC included dietary advice \\
1991 [26] & group and 71 in the control & and glycemic control \\
(USA) & group &
\end{tabular}

1991 [2

(USA)
HA1C concentration in the PCC group was lower than in the NPCC group ( $p<0.0008$ )

Spontaneous abortion rate was lower $(p<0.04)$ and there was no congenital malformations in either group.

\begin{tabular}{lll}
\hline Temple & 110 women with type I diabetes & PCC included: Glycemic \\
2006a [10] & attended the preconception & control, folic acid \\
2006b [29] & care clinic and 180 women with & supplementation, smoking \\
(UK) & type I diabetes did not attend & cessation, education.
\end{tabular}
the preconception care clinic

There was significant improvement in the outcome NPCC group in the rate of spontaneous abortion ( $p<$ between the PCC group and the $0.056)$ and in the rate of preterm delivery $(p<0.02)$. The rate of congenital malformations was lower in PCC group compared to the NPCC group ( $p$ $<0.065)$. the adverse outcome including malformations, still birth and neonatal death were significantly more in the latter group than the former one $(p<$ 0.026)

\begin{tabular}{ll}
\hline Willhoite & 62 women with either type I or \\
1993 [30] & type II diabetes who received \\
(USA) & preconception counseling and \\
& 123 women with either type I or \\
& type II diabetes who did not \\
& receive preconception \\
& counseling
\end{tabular}

PCC included counseling by PCC group had significantly less health professional the control perinatal mortality than the group received no counseling. NPCC group (OR3.9 Cl 1.2-13.9) and insignificantly less congenital malformations (OR $4.2 \mathrm{Cl} 0.5-29.7)$
Medium (52\% of preconception care patients dropped out, no blinding in the assessment of the outcome)

Low (Baseline characteristics in both groups were similar; the prospective nature of the study ascertained the completeness of the follow up, the completeness of the baseline and the outcome data. Use of appropriate statistical tests such as logistic regression analysis confirmed the association between the preconception care and outcomes).

\begin{tabular}{lll}
\hline Boulot & 172 women with either type I or & PCC included education, \\
2003 [33] & type II diabetes who received & assessment of diabetes \\
(France) & PCC and 260 women with & complications glycemic \\
& either type I or type II diabetes & control self monitoring of \\
& who did not receive PCC & blood glucose and \\
& & Contraception
\end{tabular}

Galindo

(Spain)

\section{PCC included education,} monitoring of blood glucose glycemic control self
15 women with pre-existing diabetes received PCC and 112 women with pre-existing diabetes did not receive PCC.
PCC group had significantly less perinatal mortality than the NPCC group, $(p<0.005)$ for type 1 diabetics and significantly less congenital malformations, $(p<$ 0.005) for type 1 diabetics
High (Base line characteristics of the two groups were significantly different in age, duration of diabetes and smoking all are confounding factors for the outcomes. The two groups did not receive the same antenatal intra-partum and postnatal care. The assessor of the congenital malformation was not blinded)

Low (cases and control were well defined and comparable selection bias is unlikely as consecutive cases were enrolled, the prospective nature of the study ascertained the completeness of the follow up, the completeness of the baseline and the outcome data)

The frequency of congenital abnormalities in the PCC group was $3 / 15$ compared to $14 / 112$ in the NPCC group. There was 1 spontaneous abortion in the PCC group and 9 in the group who received no PCC.

\footnotetext{
The frequency of congenital abnormalities in the PCC group was $3 / 12$ compared to $2 / 12$ in the NPCC group. In the PCC 6/ 12 neonates were macrosomic while 4/12 were macrosomic in the NPCC group. HbA1c was significantly lower in the first trimester in the PCC group compared to the NPCC group $(p<0.01)$
} Low (cases and control were well defined and comparable, selection bias is unlikely as consecutive cases were enrolled, the prospective nature of the study ascertained the completeness of the follow up, the completeness of the baseline and the outcome data)

High (Both the study population
and the control were not representative of the general diabetic population with frequency of diabetic vascular complications approaching 50\%. The PCC components were not defined neither the target blood glucose) 
Table 2 Characteristics of included retrospective cohort studies

\begin{tabular}{|c|c|c|c|c|}
\hline $\begin{array}{l}\text { Year of } \\
\text { Publication } \\
\text { (country) }\end{array}$ & Participants & Intervention v comparison & Outcome & Risk of Bias \\
\hline $\begin{array}{l}\text { Dicker } 1988 \\
\text { [15] (Israel) }\end{array}$ & $\begin{array}{l}59 \text { IDDM women attended } \\
\text { a pre-conception clinic } \\
\text { compared to } 35 \text { pregnant } \\
\text { women who did not attend }\end{array}$ & $\begin{array}{l}\text { PCC included: insulin and dietary } \\
\text { glycemic control, advice on } \\
\text { contraception and screening for } \\
\text { diabetes complications }\end{array}$ & $\begin{array}{l}\text { PCC group had significantly } \\
\text { lower HA1C at the first trimester } \\
(p<0.001) \text { and significantly } \\
\text { lower rate of spontaneous } \\
\text { abortion }(p<0.001) \text { compared } \\
\text { to the NPCC group. }\end{array}$ & $\begin{array}{l}\text { Low (Clear description of } \\
\text { participants and intervention, } \\
\text { noted confounding factors and } \\
\text { well presented results. There } \\
\text { was significant difference } \\
\text { between the two groups in the } \\
\text { diabetes complications before } \\
\text { intervention) }\end{array}$ \\
\hline $\begin{array}{l}\text { Dunne } 1999 \\
\text { [16] (UK) }\end{array}$ & $\begin{array}{l}47 \text { women with IDDM } 12 \text { of } \\
\text { them attended } \\
\text { preconception care clinic } \\
\text { and } 35 \text { women did not. }\end{array}$ & $\begin{array}{l}\text { PCC included assessment of } \\
\text { diabetes complications and } \\
\text { glycemic control }\end{array}$ & $\begin{array}{l}\text { The PCC group had significantly } \\
\text { lower level of HA1C level } \\
\text { compared to the NPCC group } \\
\text { ( } p<0.008) \text {. There were no } \\
\text { congenital malformations in } \\
\text { both groups. The cesarean } \\
\text { section rate, the macrosomia } \\
\text { rate and the small for gestation } \\
\text { age were similar between the }\end{array}$ & $\begin{array}{l}\text { Medium (Due to the audit } \\
\text { nature of the report there is no } \\
\text { clear description of the } \\
\text { intervention, some important } \\
\text { confounders were not } \\
\text { addressed such as White's } \\
\text { classification and the outcome } \\
\text { assessment was not blinded) }\end{array}$ \\
\hline
\end{tabular}
two groups

\begin{tabular}{lll}
\hline Damm 1989 & 197 attended PCC and 61 & PCC included: contraception and \\
$\begin{array}{l}\text { [14] } \\
\text { (Denmark) }\end{array}$ & didn't attend & glycemic control.
\end{tabular}

\section{The rate of congenital} malformations was significantly lower in the PPC group 1.0\% than the NPPC group $8.2 \%$, $(p<$ $0.01)$ No significant difference in subset of froption care were a the level of HA1C during the first of the study) trimester between the two group

\begin{tabular}{lll}
\hline Goldman & 44 women with type I & PCC included assessment of \\
1986 [21] & diabetes attended the & diabetic complications, \\
(Israel) & preconception clinic and 31 & Contraception advice, Glycemic \\
& women with type I diabetes & control and dietary advice \\
& did not attend
\end{tabular}

\section{The NPCC group had} significantly shorter duration of pregnancy $(p<0.05)$ significantly heavier mean birth weight $(p<0.05)$ than the PCC group. The two groups were similar in neonatal hypoglycemia, hypocalcaemia and respiratory distress syndrome

\begin{tabular}{|c|c|c|}
\hline $\begin{array}{l}\text { Fuhrmann } \\
1986[19] \& \\
1984[18] \& \\
1983[17] \\
\text { (Germany) }\end{array}$ & $\begin{array}{l}620 \text { pregnant women with } \\
\text { insulin dependent } \\
\text { diabetes, } 183 \text { received pre- } \\
\text { pregnancy care } 437 \text { women } \\
\text { did not }\end{array}$ & $\begin{array}{l}\text { PCC included: short } \\
\text { hospitalization every } 3 \text { month } \\
\text { until conception, education, self } \\
\text { monitoring of blood glucose, } \\
\text { assessment and treatment of } \\
\text { diabetes complications and } \\
\text { glycemic control }\end{array}$ \\
\hline $\begin{array}{l}\text { Rowe } 1987 \\
\text { [27] (UK) }\end{array}$ & $\begin{array}{l}21 \text { IDDM } 14 \text { received } \\
\text { preconception care and } 7 \\
\text { did not }\end{array}$ & $\begin{array}{l}\text { PCC included Glycemic control, } \\
\text { counseling and blood glucose } \\
\text { self monitoring }\end{array}$ \\
\hline
\end{tabular}

\section{PCC group had significantly} lower rate of congenital malformations $1.1 \%$ compared to the NPCC group $7.0 \%(p<$ 0.01)

High (unclear description of the participants, the intervention and the outcome, the data of the preconception care were a

Low (Clear description of participants and intervention, noted confounding factors and well presented results. There was significant difference between the two groups in the diabetes complications before intervention)

\section{Medium (Well described} intervention, no blinding for the outcome, no description of the possible confounding factors)

The PCC group had significantly High (Unclear description of the better initial HA1C level $(p<$ $0.0001)$, and lower mean birth weight $(p<0.05)$ participants, no description of possible confounding factors, no blinding in assessment of the outcome, small group, high target of $\mathrm{HbA} 1 \mathrm{C}$ 5-9\%)

\begin{tabular}{lll}
\hline Steel 1990 & 143 IDDM women attended & PCC included: education, \\
[28] \& 1982 & the preconception care & glycemic controlled and \\
[31] (UK- & clinic and 96 IDDM women & contraception \\
Scotland) & did not attend &
\end{tabular}

\author{
PCC group had lower initial \\ $\mathrm{HbA1C}$ as compared to NPCC \\ group $(p<0.0001)$ and lower \\ rate of congenital mal \\ formations $(p<0.005)$, \\ maternal hypoglycemia was \\ significantly common in the \\ PCC group than the NPCC ( $p<$ \\ 0. 001)
}


Table 3 Characteristics of included case-control studies

\begin{tabular}{|c|c|c|c|c|}
\hline $\begin{array}{l}\text { Study/Year } \\
\text { of } \\
\text { Publication } \\
\text { (country) }\end{array}$ & Participants & Intervention & Outcome & Risk of Bias/Notes \\
\hline $\begin{array}{l}\text { Correa } \\
2003 \text { [13] } \\
\text { USA }\end{array}$ & $\begin{array}{l}\text { Cases were } 3278 \text { Infants with } \\
\text { congenital malformations related to } \\
\text { diabetes. Controls were } 3029 \text { infants } \\
\text { without congenital malformations. } \\
\text { Maternal diabetes and intake of } \\
\text { multivitamin were evaluated as a risk } \\
\text { factors for congenital malformations }\end{array}$ & $\begin{array}{l}\text { PCC included the } \\
\text { use of } \\
\text { multivitamin for } \\
3 \text { month before } \\
\text { conception }\end{array}$ & $\begin{array}{l}\text { The risk of congenital malformations } \\
\text { related to diabetes was limited to } \\
\text { infants of } \mathrm{f} \text { diabetic mothers who had } \\
\text { not taken multivitamin (OR } 3.3995 \% \\
\mathrm{Cl} 1.79-8.63 \text { ). Mother who had taken } \\
\text { multivitamin had no increase risk of } \\
\text { congenital malformations related to } \\
\text { diabetes (OR } 0.1595 \% \mathrm{Cl} 0.00-1.99 \text { ) }\end{array}$ & $\begin{array}{l}\text { Medium (clear definition and } \\
\text { selection of cases and controls, and } \\
\text { outcomes, clearly defined outcome, } \\
\text { not clear if the interviewers were } \\
\text { blinded to the outcome, recall bias } \\
\text { cannot be excluded during the } \\
\text { interviews) }\end{array}$ \\
\hline
\end{tabular}

Key: OR= Odd Ratio, $\mathrm{Cl}=$ Confidence Interval.

The evidence did not support the effectiveness of the PCC in reducing, spontaneous abortion, preeclampsia, cesarean delivery, macrosomia, RDS, SGA and neonatal hypoglycemia (Table 5 and Additional file 1:Appendix 3).

The use of multivitamins in the preconception period as a sole intervention, was evaluated by one case control study [13] and was found not to be effective in reducing the rate of congenital malformations (Odd Ratio (OR) 0.15 95\% CI 0.00-1.99).

Similarly one study, at high risk of bias, evaluated the effectiveness of preconception counseling, as a sole intervention, in improving fetal and neonatal outcomes, showed improvement in total mortality (still birth and neonatal death) and the rate of congenital malformation [30].

Hypoglycemia as adverse effect of PCC was evaluated by two studies $[10,28]$. Meta-analysis of the pooled data did not show difference between the PCC and the control group (Figure 7).

Data were not available for the evaluation of the effects of PCC on polyhydramninos, termination of pregnancy for congenital malformations, induction of labor, birth trauma, shoulder dystocia and admission to NICU.

\section{Discussion}

Our systematic review of the effectiveness of PCC in the improvement of maternal and fetal outcomes, found sufficient evidence to support its implementation in practice.

The nature of the intervention lent strength to the observational studies by avoiding certain biases known to occur in such study designs. Lack of allocation concealment and blinding of participants were avoided by recruiting the intervention and the control groups at different times during the course of the study (preconception period and antenatal period). Due to the relatively short duration of the pregnancy, attrition bias was noted in only one study, [26] all other studies had complete follow up of both groups. However the problem of confounding factors such as smoking, maternal age, parity and vascular complications of diabetes, was noted by most of the studies but only one study used the appropriate statistical test to quantify the effect of the PCC apart from the confounders [10].

The homogeneity of the participants, the intervention and the outcomes gives confidence in the estimated effects of the PCC from the pooled data.

The effectiveness of PCC in reducing congenital malformations is impressive (Table 5 and Figure 2) and has practical implication considering the recent report of the $\mathrm{CEMCH}$ [4] which showed that congenital malformations rate in infants of diabetic mothers in England, Wales and Northern Ireland is more than twice the background population rate. This finding is also of a paramount

Table 4 Characteristics of included controlled trials

\begin{tabular}{|c|c|c|c|c|}
\hline $\begin{array}{l}\text { Study/Year of Publication } \\
\text { (country) }\end{array}$ & Participants & $\begin{array}{l}\text { Intervention } \\
\mathbf{v} \\
\text { comparison }\end{array}$ & Outcome & Risk of Bias/Note \\
\hline $\begin{array}{l}\text { Pregnancy outcome in } \\
\text { Diabetes control and } \\
\text { complication trial research } \\
\text { group } 1996 \text { [12] (USA) }\end{array}$ & $\begin{array}{l}187 \text { had } \\
\text { preconception } \\
\text { intensive insulin } \\
\text { therapy and } 83 \\
\text { did not. }\end{array}$ & $\begin{array}{l}\text { PCC included } \\
\text { glycemic } \\
\text { control and } \\
\text { dietary advice. }\end{array}$ & $\begin{array}{l}\text { There were } 26 \text { spontaneous abortion in } \\
\text { the PCC group and } 16 \text { in the NPCC } \\
\text { group. One still birth in the PCC group } \\
\text { and } 3 \text { in the NPCC. Congenital mal } \\
\text { formations were } 5 \text { in the PCC group and } 4 \\
\text { in the NPCC group. No differences on } \\
\text { neonatal morbidity or maternal } \\
\text { morbidity. Mean HbA1C in PCC group } \\
=7.4 \pm 1.3 \text { and in NPCC }=8.8 \pm 1.7\end{array}$ & $\begin{array}{l}\text { High (Unclear report of the outcome, } \\
\text { the control group was aware of the } \\
\text { importance of glycemic control and } \\
\text { was repeatedly advised to change into } \\
\text { intensive therapy when planning } \\
\text { pregnancy. So intervention was not } \\
\text { restricted to the preconception group. } \\
\text { No specific target level of the blood } \\
\text { sugar was stated for the preconception } \\
\text { group) }\end{array}$ \\
\hline
\end{tabular}


Table 5 Pooled estimates effect of preconception care

\begin{tabular}{lll}
\hline Dichotomous outcomes of preconception care & No of studies [references] & Risk Ratio (95\%Confedance interval) \\
\hline Congenital malformation & $11[10,16,19-21,23,25,26,28,32,33]$ & $0.25(0.15,0.42)$ \\
\hline Perinatal Mortality & $5[10,16,20,23,33]$ & $0.35(0.15,0.82)$ \\
\hline Macrosomia & $3[10,20,23]$ & $1.03(0.81,1.30)$ \\
\hline Cesarean Section & $5[10,16,20,21,23]$ & $1.08(0.96,1.22)$ \\
\hline Preterm Delivery & $4[10,16,20,23]$ & $0.7(0.55,0.90)$ \\
\hline Pre-eclampsia & $3[10,20,21]$ & $0.92(0.62,1.35)$ \\
\hline Neonatal Hypoglycemia & $3[20,21,23]$ & $0.65(0.39,1.08)$ \\
\hline Maternal Hypoglycemia & $2[10,28]$ & $1.51(1.15,1.99)$ \\
\hline Spontaneous Abortion & $7[10,15,20,23,25,26,32]$ & $0.78(0.55,1.11)$ \\
\hline Respiratory Distress Syndrome & $3[20,21,23]$ & $0.55(0.26,1.16)$ \\
\hline Small for Gestation Age & $2[20,23]$ & $0.26(0.05,1.41)$ \\
\hline Continuous outcomes & Number of studies ( references) & Means difference (95\% Cl) \\
\hline The difference in the level of glycosylated Hemoglobin A1C & $4[10,21,26,28]$ & $2.43(2.27,2.58)$ \\
\hline The difference in gestational age at first visit to antenatal care & $3[10,23,26]$ & $1.32(1.23,1.40)$ \\
\hline
\end{tabular}

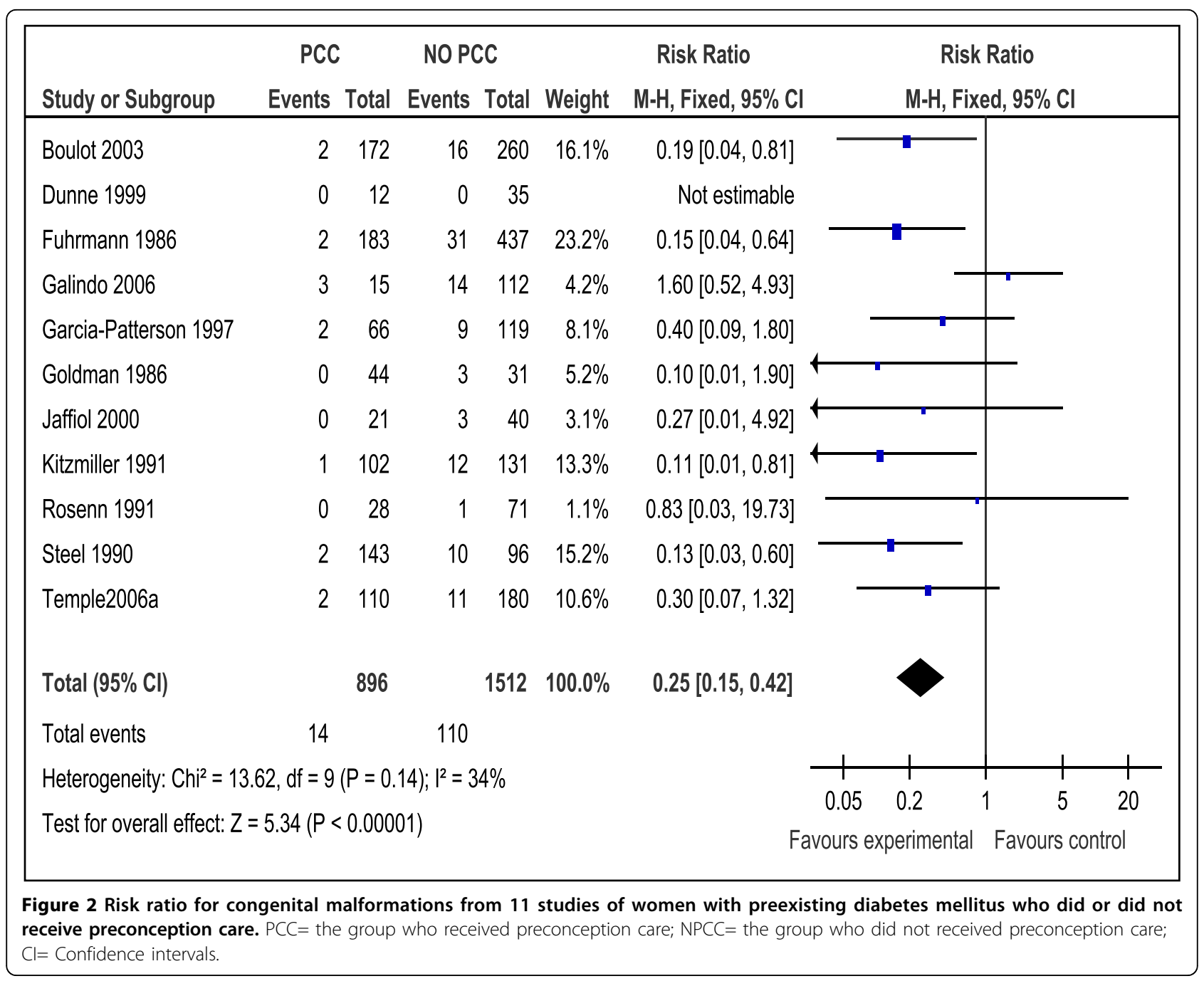




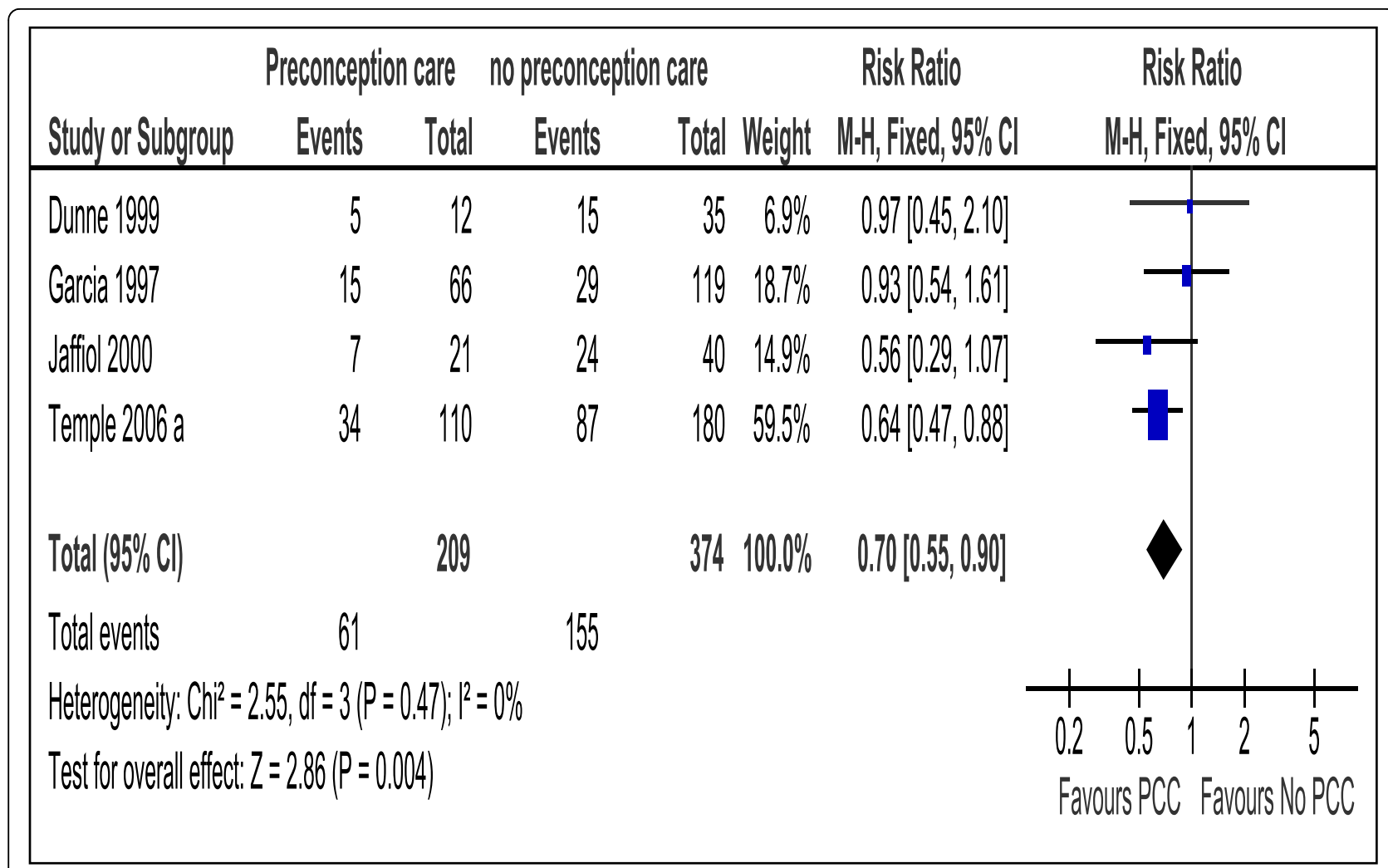

Figure 3 Risk ratio for preterm delivery from 4 studies of women with preexisting diabetes mellitus who did or did not receive preconception care. $\mathrm{PCC}=$ Preconception care; NPCC= No preconception care; $\mathrm{Cl}=$ Confidence intervals.

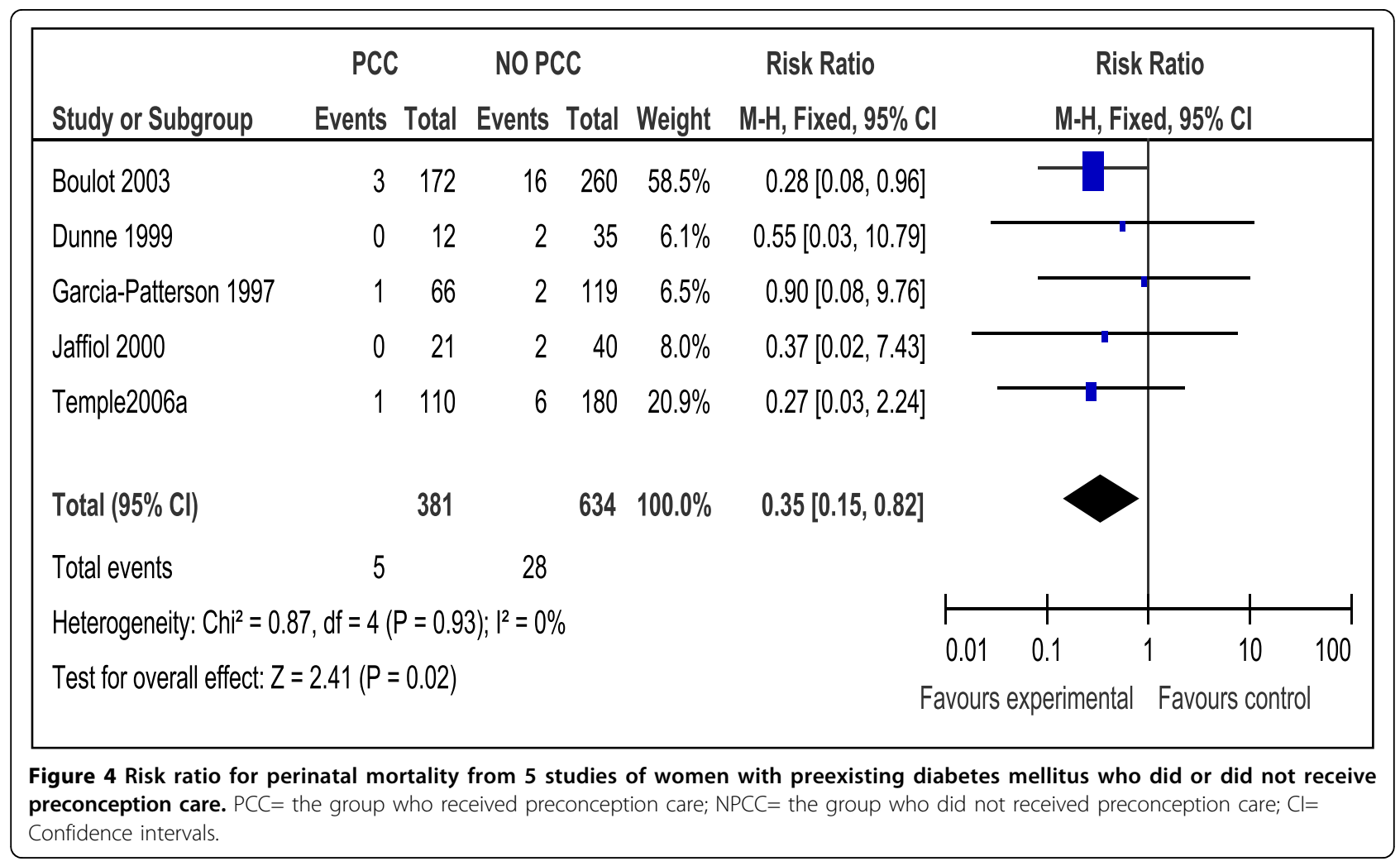




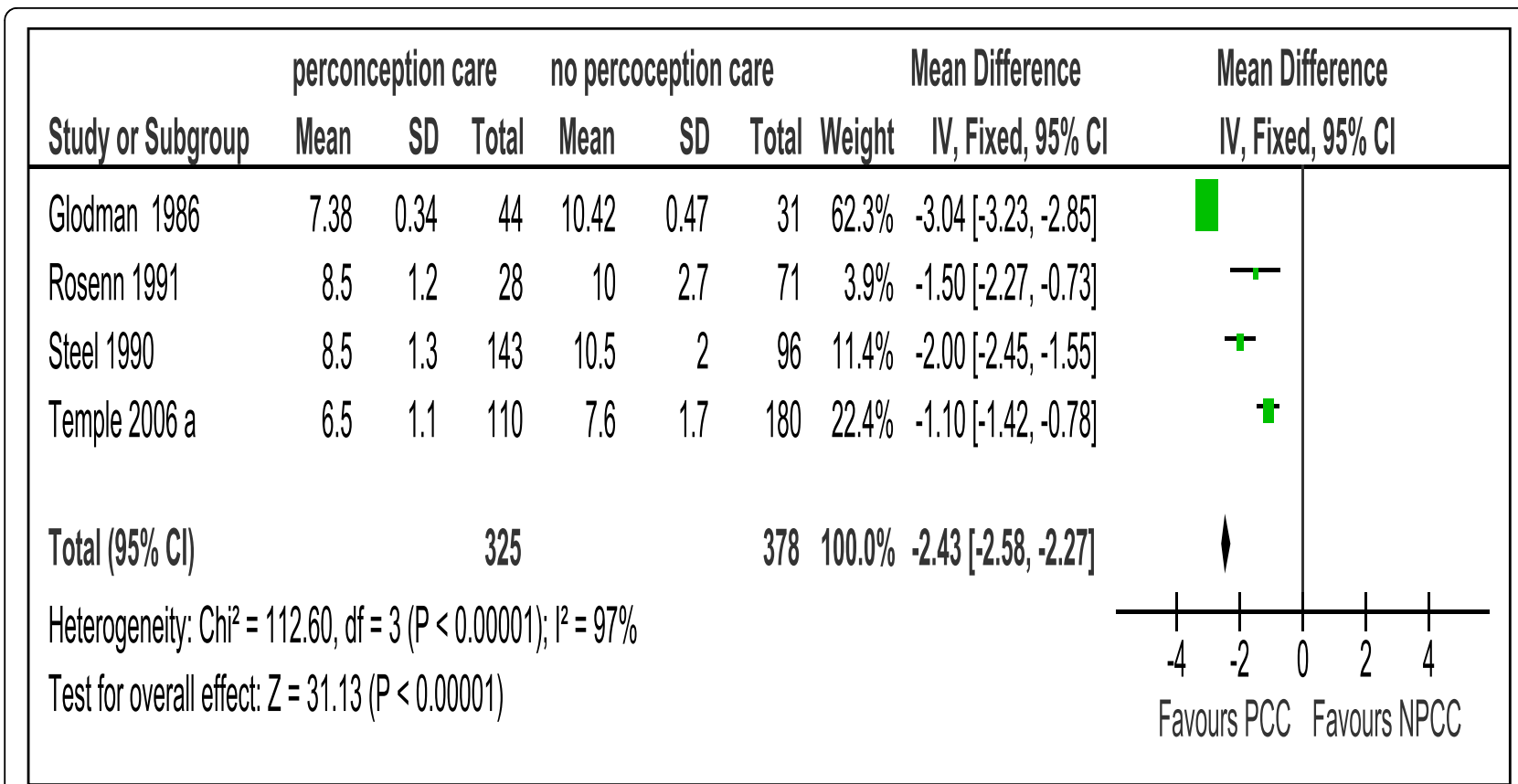

Figure 5 First trimester mean value of glycosylated hemoglobin from 4 studies of women with preexisting diabetes mellitus who did or did not receive preconception care. $\mathrm{PCC}=$ Preconception care; NPCC= No preconception care; $\mathrm{Cl}=$ Confidence intervals

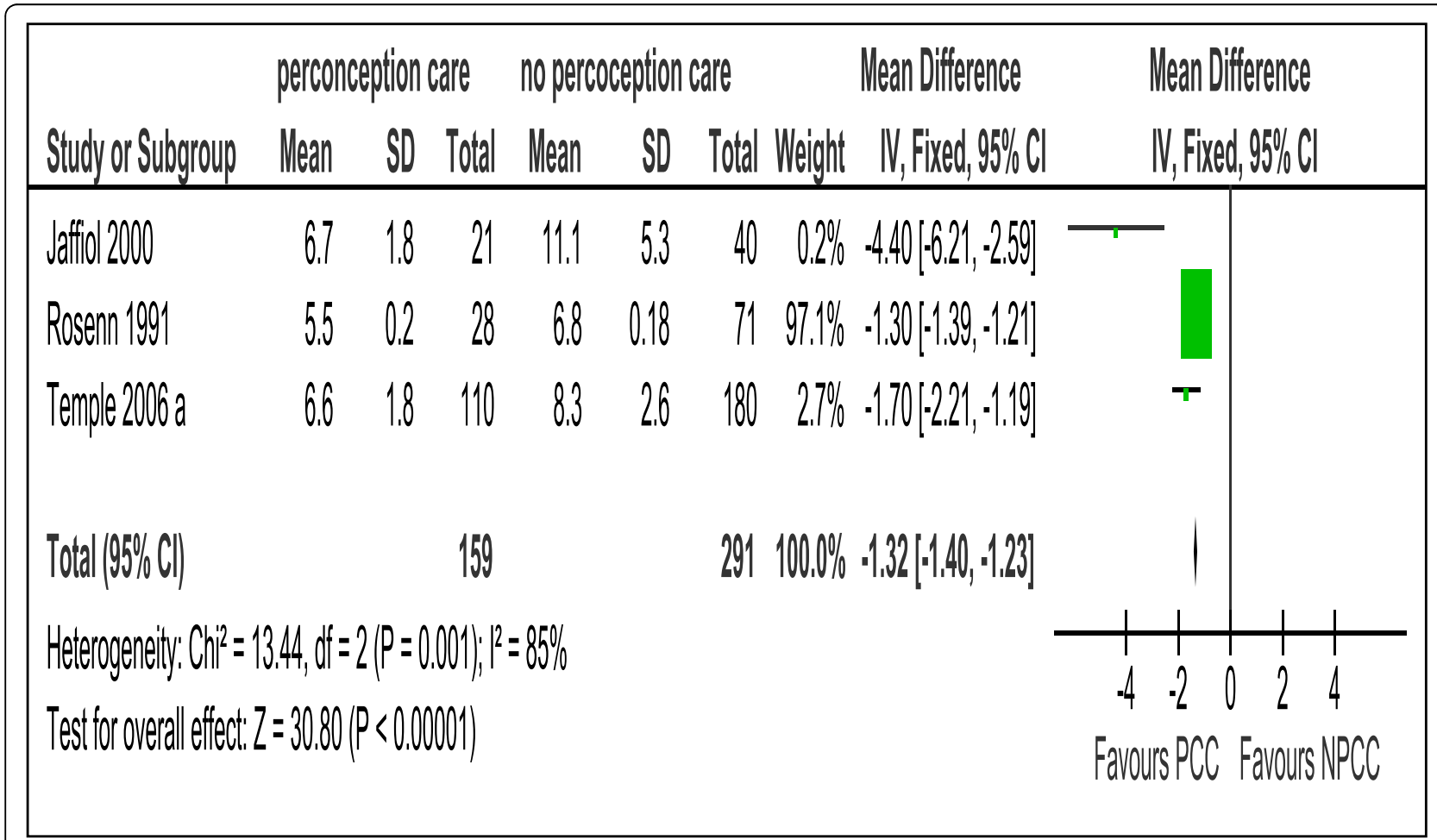

Figure 6 The mean gestation age at the time of the first antenatal visit from 3 studies of women with preexisting diabetes mellitus who did or did not receive preconception care. $\mathrm{PCC}=$ Preconception care; NPCC= No preconception care; Cl= Confidence intervals. 


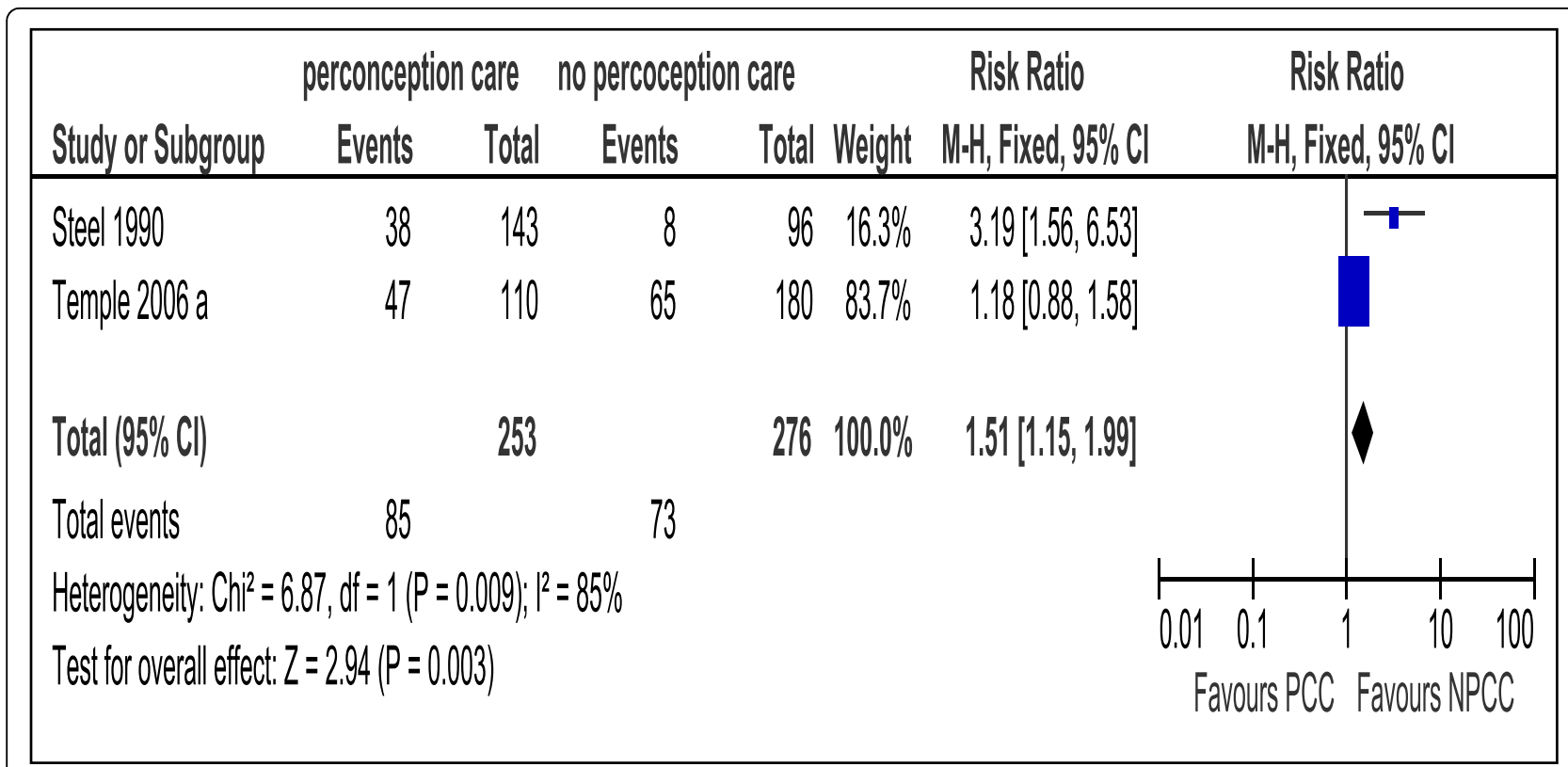

Figure 7 Risk ratio for maternal hypoglycemia from 2 studies of women with preexisting diabetes mellitus who did or did not receive preconception care. $\mathrm{PCC}=$ Preconception care; $\mathrm{NPCC}=$ No preconception care; $\mathrm{Cl}=$ Confidence intervals.

importance to many communities in the Middle East [35], North Africa [36] and some communities in Asia [37] where the burden of congenital malformation is very high due to many causes including maternal diabetes.

The effect of PCC in reducing the rate of congenital malformations reflected positively on its effect in reducing the perinatal mortality among women who utilized the care (Figure 4). This effect addresses a major health problem of four folds increase in the perinatal mortality in mothers with preexisting diabetes when compared to the general population [38]

The meta-analysis supported the effectiveness of the PCC in reducing the rate of preterm delivery (Table 5 and Figure 3). We believe that effect would have been larger if data were available for very preterm delivery $\leq 34$ weeks of gestation when the effect of the preconception rather than the antenatal care is evaluated as demonstrated by one study [10].

Maternal hyperglycemia during the period of organogenesis is known to be associated with congenital malformations $[39,40]$. The analysis of the pooled data in this review suggested that PCC is effective in reducing the level of HbA1C during the first trimester of pregnancy and hence the risk of congenital malformations (Table 5 and Figure 5).

We were surprised that meta-analysis did not support the effectiveness of PCC in improving the rates of spontaneous abortion (Table 5 and Additional file 1: Appendix 3). We suggest that this result is due to late attendance of the control group for antenatal care by which time some events of spontaneous abortion might have been missed. This suggestion was further supported by meta-analysis of the gestation age at first visit for the PCC and the control groups, (Figure 6) which showed significant difference between the two groups.

In this review one case control study addressed the effectiveness of multivitamins supplementation in the preconception period, as an isolated intervention, in reducing the rate of congenital malformations[13]. The role of folic acid and multivitamins in the prevention of some congenital malformation is well documented [41]. However all other studies included in this review, except for one recent report [10], did not include multivitamin or folic acid in their program of PCC, which supports an expectation of larger effect of PCC in improving fetal and neonatal outcomes if folic acid or multivitamin supplementation becomes an integral part of that care.

Another isolated preconception intervention proved to be effective in improving fetal and neonatal outcomes, is women counseling, an intervention evaluated by only one study [30]

Other outcomes which did not improve by PCC, such as pre-eclampsia, cesarean delivery and macrosomia (Table 5 and Additional file 1: Appendix 3), might be related to care during the latter part of pregnancy rather than the preconception period. However few studies were included in the meta-analysis for these outcomes and further larger studies, with more participants might prove the effectiveness of PCC in improving some or all of these outcomes. 
Only two studies evaluated maternal hypoglycemia as adverse effect of PCC $[10,28]$ and the pooled data showed no difference between the two groups (Figure 7). Marked heterogeneity might be due to the differences in the target blood glucose level between the two studies.

One study conducted an economic evaluation of PCC and found that it is associated with considerable saving and reduced resources utilization [22] and yet population based studies showed that only $34-38 \%$ of eligible women receive PCC $[4,30]$.

We suggest that more research is needed in methods of encouraging diabetic women to utilize PCC.

Our review confirms previous findings by Ray et al [9]. The strength of our review comes from the comprehensive evaluation of the available evidence on the effectiveness and safety of PCC in improving maternal and fetal outcomes together with assessment of wide range of interventions which we considered as PCC and all the possible maternal, fetal or neonatal outcomes which are affected by maternal preexisting DM. However we are aware of the limitation of the observations studies as the sole source of evidence and the inherent bias associated with the design of the cohort studies included in the meta-analysis.

The review carries important implications for practice and research as it highlights the importance of the integration of PCC in the routine care of diabetic women during their reproductive age.

\section{Conclusion}

PCC for women with preexisting type 1 or type $2 \mathrm{DM}$ is effective in improving rates of congenital malformation, perinatal mortality, preterm labour, level of maternal HbA1c in the first trimester of pregnancy and maternal early utilization of antenatal care.

\section{Additional material}

Additional files 1: Appendices Appendix 1: Search Strategy (the systematic review search strategy). Appendix 2: Table of excluded studies (description of studies excluded from the review and the reasons for exclusion). Appendix 3: maternal and fetal outcomes not improved by preconception care (Forest Plots of meta-analysis of outcomes which are not improved by preconception care).

\section{Acknowledgements}

We acknowledge the help of Vittoria Lutje in designing the search strategy and in conducting the search for this review and the help of Dr Thomas Jefferson in reviewing the manuscript.

\section{Authors' contributions}

HW conceived the idea of the review, was responsible for drafting and writing the study protocol and reviewing the search strategy. HW, RZ, GB, LA and SE were responsible for study selection and data extraction. HW and RZ were responsible for quality assessment of studies and data analysis. HW was responsible for writing the final report. LA, HW, RZ, GB and SE reviewed and approved the final manuscript.

\section{Competing Interests}

The authors declare that they have no conflict of interest.

Received: 15 March 2010 Accepted: 14 October 2010

Published: 14 October 2010

\section{References}

1. Wild S, Roglic G, Green A, Sicree R, King H: Global prevalence of diabetes: estimates for the year 2000 and projections for 2030. Diabetes Care 2004, 27:1047-1053.

2. Al-Nuaim AR: Prevalence of glucose intolerance in urban and rural communities of Saudi Arabia. Diabetic Medicine 1997, 14:595-602.

3. Hotu S, Carter B, Watson PD, Cutfield WS, Cundy T: Increasing prevalence of type 2 diabetes in adolescents. journal of paediatric and child health 2004, 40:201-204.

4. Confidential Enquiry into Maternal and Child Health (CEMACH). Pregnancy in Women with type 1 and type 2 Diabbetes in 2002-2003. England Wales and Northern Ireland. London. CEMACH 2005.

5. Casson IF, Clarke CA, Howard CV, McKendrick O, Pennycook S, Pharonah $\mathrm{PO}$, et al: Outcomes of pregnancy in insulin dependent diabetic women:result of a five year population cohort study. BMJ 1997 315:275-278.

6. Yang J, Cummings EA, O'Connell C, Jangaard K: Fetal and Neonatal Outcomes of Diabetic Pregnancies. Obstetrics and Gynecology 2006, 108:644-650.

7. Diabetes Care and Research in Europe. The Saint Vincent Declaration. Diabet Med 1990, 7.

8. Sobande AA, Al-Bar H, Archibong E: Diabetes and Preinatal Loss. A continuing Problem. Saudi Med J 2000, 21:161-163.

9. Ray JG, O'Brien TE, Chan WS: Preconception care and the risk of congenital anomalies in the offspring of women with diabetes mellitus: a meta-analysis. QJM 2001, 94:435-444.

10. Temple RC, Aldridge VJ, Murphy HR: Prepregnancy care and pregnancy outcomes in women with type 1 diabetes. Diabetes Care 2006, 29:1744-1749.

11. Well GA, Shea B, C'Connell D, Peterson J, Welch V, Losos M, et al: The Newcaslte-Ottawa Scal (NOS) for assessing the quality of nonrandomised studies in met-analyses. 2005 [http://www.ohri.ca/ programs/clinical_epidemiology/oxford.asp].

12. Pregnancy outcomes in the Diabetes Control and Complications Trial. Am J Obstet Gynecol 1996, 174:1343-1353.

13. Correa A, Botto L, Liu Y, Mulinare J, Erickson JD: Do multivitamin supplements attenuate the risk for diabetes-associated birth defects? Pediatrics 2003, 111:1146-1151.

14. Damm $P$, Molsted-Pedersen L: Significant decrease in congenital malformations in newborn infants of an unselected population of diabetic women. Am J Obstet Gynecol 1989, 161:1163-1167.

15. Dicker D, Feldberg D, Samuel N, Yeshaya A, Karp M, Goldman JA: Spontaneous abortion in patients with insulin-dependent diabetes mellitus: the effect of preconceptional diabetic control. Am J Obstet Gynecol 1988, 158:1161-1164.

16. Dunne FP, Brydon P, Smith T, Essex M, Nicholson H, Dunn J: Preconception diabetes care in insulin-dependent diabetes mellitus. QJM 1999, 92:175-176.

17. Fuhrmann $\mathrm{K}$, Reiher $\mathrm{H}$, Semmler $\mathrm{K}$, Fischer F, Fischer M, Glockner E: Prevention of congenital malformations in infants of insulin-dependent diabetic mothers. Diabetes Care 1983, 6:219-223.

18. Fuhrmann K, Reiher H, Semmler K, Glockner E: The effect of intensified conventional insulin therapy before and during pregnancy on the malformation rate in offspring of diabetic mothers. Experimental and Clinical Endocrinology 1984, 83:173-177.

19. Fuhrmann K: Treatment of pregnant insulin-dependent diabetic women. Acta Endocrinol Suppl (Copenh) 1986, 277:74-76.

20. Garcia-Patterson A, Corcoy R, Rigla M, Caballero A, Adelantado JM, Altirriba $\mathrm{O}$, et al: Does preconceptional counselling in diabetic women influence perinatal outcome? Ann Ist Super Sanita 1997, 33:333-336.

21. Goldman JA, Dicker D, Feldberg D, Yeshaya A, Samuel N, Karp M: Pregnancy outcome in patients with insulin-dependent diabetes 
mellitus with preconceptional diabetic control: a comparative study. Am J Obstet Gynecol 1986, 155:293-297.

22. Herman WH, Janz NK, Becker MP, Charron-Prochownik D: Diabetes and pregnancy. Preconception care, pregnancy outcomes, resource utilization and costs. J Reprod Med 1999, 44:33-38.

23. Jaffiol C, Baccara MT, Renard E, Apostol DJ, Lefebvre P, Boulot P, et al: [Evaluation of the benefits brought by pregnancy planning in type 1 diabetes mellitus]. Bull Acad Natl Med 2000, 184:995-1007.

24. Jensen BM, Kuhl C, Molsted-Pedersen L, Saurbrey N, Fog-Pedersen J: Preconceptional treatment with insulin infusion pumps in insulindependent diabetic women with particular reference to prevention of congenital malformations. Acta Endocrinol Suppl (Copenh) 1986, 277:81-85.

25. Kitzmiller $\mathrm{L}$, Gavin LA, Gin GD, Jovanovic-Peterson L, Main EK, Zigrang WD: Preconception care of diabetes. Glycemic control prevents congenital anomalies. JAMA 1991, 265:731-736.

26. Rosenn B, Miodovnik M, Combs CA, Khoury J, Siddiqi TA: Pre-conception management of insulin-dependent diabetes: improvement of pregnancy outcome. Obstet Gynecol 1991, 77:846-849.

27. Rowe BR, Rowbotham CJ, Barnett AH: Pre-conception counselling, birth weight, and congenital abnormalities in established and gestational diabetic pregnancy. Diabetes Res 1987, 6:33-35.

28. Steel JM, Johnstone FD, Hepburn DA, Smith AF: Can prepregnancy care of diabetic women reduce the risk of abnormal babies? BMJ 1990, 301:1070-1074.

29. Temple RC, Aldridge V, Stanley K, Murphy HR: Glycaemic control throughout pregnancy and risk of pre-eclampsia in women with type I diabetes. BJOG 2006, 113:1329-1332.

30. Willhoite MB, Bennert HW Jr, Palomaki GE, Zaremba MM, Herman WH, Williams JR, et al: The impact of preconception counseling on pregnancy outcomes. The experience of the Maine Diabetes in Pregnancy Program. Diabetes Care 1993, 16:450-455.

31. Steel JM, Johnstone FD, Smith AF, Duncan LP: Five years' experience of "prepregnancy" clinic for insulin-dependent diabetics. BMJ 285:353-356, 82 A.D.

32. Galindo A, Burguillo AG, Azriel S, Fuente PL: Outcome of fetuses in women with pregestational diabetes mellitus. J Perinat Med 2006, 34:323-331.

33. Boulot $P$, Chabbert-Buffet $N$, d'Ercole C, Floriot $M$, Fontaine $P$, Fournier $A$, et al: French multicentric survey of outcome of pregnancy in women with pregestational diabetes. Diabetes Care 2003, 26:2990-2993.

34. Garcia Ingelmo MT, Herranz dIM, Martin VP, Janez FM, Grande AC, Pallardo Sanchez LF: [Preconceptional control in diabetic women]. Rev Clin Esp 1998, 198:80-84.

35. Al-Gazali L, Hamamy H, Al-Arrayad S: Genetic disorders in the Arab world. BMJ 2006, 333:831-834.

36. Jaouad IC, Elalaoui SC, Sbiti A, Elkerh F, Belmahi L, Sefiani A: Consanguineous marriages in Morocco and the consequence for the incidence of autosomal recessive disorders. J Biosoc Sci 2009, 41:575-581.

37. Hussain $\mathrm{R}$, Bittles $A H$, Sullivan $\mathrm{S}$ : Consanguinity and early mortality in the Muslim populations of India and Pakistan. Am J Hum Biol 2001, 13:777-787.

38. Casson IF: Pregnancy in women with diabetes - After the CEMACH report, what now? Diabetic Medicine 2006, 23:481-484.

39. Corrigan N, Brazil DP, McAuliffe F: Fetal cardiac effects of maternal hyperglycemia during pregnancy. Birth Defects Res A Clin Mol Teratol 2009, 85:523-530.

40. Riskin-Mashiah S, Younes G, Damti A, Auslender R: First-trimester fasting hyperglycemia and adverse pregnancy outcomes. Diabetes Care 2009, 32:1639-1643.

41. Czeizel AE: Primary prevention of neural-tube defects and some other major congenital abnormalities: recommendations for the appropriate use of folic acid during pregnancy. Paediatr Drugs 2000, 2:437-449.

\section{Pre-publication history}

The pre-publication history for this paper can be accessed here: http://www.biomedcentral.com/1471-2393/10/63/prepub

doi:10.1186/1471-2393-10-63

Cite this article as: Wahabi et al:: Preconception care for diabetic women for improving maternal and fetal outcomes: a systematic review and meta-analysis. BMC Pregnancy and Childbirth 2010 10:63.

\section{Submit your next manuscript to BioMed Central and take full advantage of:}

- Convenient online submission

- Thorough peer review

- No space constraints or color figure charges

- Immediate publication on acceptance

- Inclusion in PubMed, CAS, Scopus and Google Scholar

- Research which is freely available for redistribution 